\title{
The expression of a recombinant glycolate dehydrogenase polyprotein in potato (Solanum tuberosum) plastids strongly enhances photosynthesis and tuber yield
}

\author{
Greta Nölke ${ }^{1}$, Marcel Houdelet ${ }^{1}$, Fritz Kreuzaler ${ }^{1}$, Christoph Peterhänsel ${ }^{2}$ and Stefan Schillberg ${ }^{1,3, *}$ \\ ${ }^{1}$ Fraunhofer Institute for Molecular Biology and Applied Ecology IME, Aachen, Germany \\ ${ }^{2}$ Institute of Botany, Leibniz-University Hannover, Hannover, Germany \\ ${ }^{3}$ Phytopathology Department, Institute for Phytopathology and Applied Zoology, Justus-Liebig University Giessen, Giessen, Germany
}

Received 27 October 2013;

revised 7 January 2014;

accepted 3 February 2014

*Correspondence (Tel +49 2416085 11050;

fax +492416085 10000;

email stefan.schillberg@ime.fraunhofer.de)

Keywords: biomass, carbon metabolism, photorespiration, transgenic plants, tuber size.

\begin{abstract}
Summary
We have increased the productivity and yield of potato (Solanum tuberosum) by developing a novel method to enhance photosynthetic carbon fixation based on expression of a polyprotein (DEFp) comprising all three subunits ( $D, E$ and $F$ ) of Escherichia coli glycolate dehydrogenase $(G \mid c D H)$. The engineered polyprotein retained the functionality of the native GlcDH complex when expressed in E. coli and was able to complement mutants deficient for the $D, E$ and $F$ subunits. Transgenic plants accumulated DEFp in the plastids, and the recombinant protein was active in planta, reducing photorespiration and improving $\mathrm{CO}_{2}$ uptake with a significant impact on carbon metabolism. Transgenic lines with the highest DEFp levels and GlcDH activity produced significantly higher levels of glucose (5.8-fold), fructose (3.8-fold), sucrose (1.6-fold) and transitory starch (threefold), resulting in a substantial increase in shoot and leaf biomass. The higher carbohydrate levels produced in potato leaves were utilized by the sink capacity of the tubers, increasing the tuber yield by 2.3 -fold. This novel approach therefore has the potential to increase the biomass and yield of diverse crops.
\end{abstract}

\section{Introduction}

Potato is an important nongrain food commodity, with global production exceeding 373 million tonnes in 2011 (FAOSTAT, 2012). It is the primary staple food for more than one billion people worldwide, reflecting its high nutritional value, adaptability to diverse environments and potential yield (Wang, 2008). The tuber starch content also makes potato a versatile and sustainable raw material for the starch and biofuel industries. The additive effects of population growth, the emerging bioenergy economy and the loss of agricultural land to urbanization and land degradation means that greater agricultural productivity is required per hectare of land to meet the demands for food, feed, biomaterials and biofuel (FAO, 2009; OECD/FAO, 2011).

One way to increase the productivity of potato crops is to enhance their photosynthetic efficiency, because carbon metabolism in higher plants drives growth and helps to determine the yield (Long et al., 2006). Previous studies have shown that carbon metabolism can be optimized by genetic engineering (Peterhansel et al., 2008). Enhanced photosynthesis and biomass production has been achieved in tobacco by overexpressing the Calvin cycle enzyme SBPase/FBPase (Lefebvre et al., 2005; Miyagawa et al., 2001). Therefore, enhancing the efficiency of photosynthesis, and thus the amount of fixed carbon could be a straightforward approach to boost the productivity of important agronomic crops.

In C3 plants, photorespiration metabolizes the products of the ribulose-1,5-biphosphate (RuBisCO) oxygenation reaction, and thus reduces the efficiency of photosynthesis by removing carbon from the Calvin cycle, nitrogen and consuming reducing power (reviewed by Maurino and Peterhansel, 2010; Peterhansel and Maurino, 2011). Although the RuBisCO carboxylation reaction produces two molecules of phosphoglycerate that enter the Calvin cycle (ultimately to produce glucose, sucrose and starch and to regenerate ribulose-1,5-biphosphate), the oxygenation reaction produces single molecules of phosphoglycerate and phosphoglycolate, the latter being converted to phosphoglycerate by photorespiration (Leegood et al., 1995; Tolbert, 1997). The balance between these two activities depends mainly on the $\mathrm{CO}_{2} / \mathrm{O}_{2}$ ratio in the leaves (Laing et al., 1974). Abiotic stress, such as drought and heat, increase the rate of photorespiration. Although photorespiration is necessary for plant growth and survival (Peterhansel and Maurino, 2011), it is an inefficient and wasteful process that lowers the overall efficiency of photosynthesis by removing $25 \%$ of the fixed carbon and reduced nitrogen (Wingler et al., 2000). Therefore, reducing metabolic flux through the photorespiration pathway should increase resource-use efficiency, promote growth and increase yields.

The first attempts to increase yields by inhibiting photorespiration were unsuccessful because the photorespiration mutant phenotype was lethal under ambient $\mathrm{CO}_{2}$ conditions (Somerville, 1984; Somerville, 2001). This is because photorespiration is necessary to protect plants from the accumulation of toxic photosynthesis inhibitors (Campbell and Ogren, 1990; Givan and Kleczkowski, 1992), the effects of excess light (Kozani and Takeba, 1996; Wingler et al., 2000) and to provide reducing 
power for nitrate assimilation (Bloom et al., 2010; Rachmilevitch et al., 2004).

Photorespiration is an inevitable consequence of the RuBisCO catalytic mechanism, as $\mathrm{O}_{2}$ and $\mathrm{CO}_{2}$ compete for the same active site on the RuBisCO enzyme, but its impact on the plant can be reduced by engineering a photorespiratory bypass in the chloroplast (Kebeish et al., 2007; reviewed by Peterhansel et al., 2012), or by the complete oxidation of glycolate to $\mathrm{CO}_{2}$ in the chloroplast (Fahnenstich et al., 2008; Maier et al., 2012). Stepwise transformation has been used to introduce the bacterial glycolate catabolic pathway into Arabidopsis thaliana chloroplasts to convert glycolate into glycerate (Kebeish et al., 2007). The plants were sequentially transformed with genes encoding the three subunits of glycolate dehydrogenase (D, E and F), glyoxylate carboxyligase $(\mathrm{G})$ and tartronic semialdehyde reductase $(\mathrm{T})$. The resulting transgenic plants showed higher levels of net photosynthesis and produced more shoot and root biomass. Similar results were achieved when only the genes encoding glycolate dehydrogenase (DEF), the first enzyme in the pathway, were introduced.

Here, we tested the ability of Escherichia coli glycolate dehydrogenase to increase photosynthetic efficiency and biomass accumulation in potato, which is an ideal model because of its high source-to-sink capacity. The three subunits of $E$. coli glycolate dehydrogenase are expressed at different levels (Pellicer et al., 1996), and they assemble to form a functional GlcDH enzyme. The introduction of GlcDH into plants therefore typically requires multiple gene transfer. Data from the transgenic Arabidopsis thaliana plants mentioned above showed that the relative scarcity of the $\mathrm{F}$ subunit could limit the assembly of the complete DEF complex (Kebeish et al., 2007). The three corresponding bacterial genes $(g / c D, g / c E$ and $g / c F)$ were therefore fused to create a polyprotein construct with intervening flexible linkers. The activity of the engineered GlcDH polyprotein (DEFp) was initially verified in $E$. coli, and then by expression in the plastids of potato plants to test the impact of DEFp on carbon metabolism, photosynthetic efficiency and biomass accumulation.

\section{Results and Discussion}

\section{DEFp displays glycolate dehydrogenase activity in $E$. coli}

The $D, E$ and $F$ subunits of $E$. coli glycolate dehydrogenase have previously been introduced step-wise into Arabidopsis thaliana plants to reduce the loss of fixed carbon and nitrogen during photorespiration (Kebeish et al., 2007). However, to avoid the time-consuming and cumbersome process of multigene transformation, we designed a recombinant glycolate dehydrogenase polyprotein (DEFp) by fusing the corresponding E. coli glcD (1493 bps), g/cE (1046 bps) and g/cF (1220 bps) cDNAs with intervening flexible $\left(\mathrm{Gly}_{4} \mathrm{Ser}\right)_{3}$ linkers (Figure 1a). This strategy ensured that the three subunits were expressed in stoichiometric amounts, which is not always possible when separate transgenes are expressed (Kebeish et al., 2007). Prior to plant transformation, the engineered DEFp construct was overexpressed in E. coli allowing us to carry out rapid glycolate dehydrogenase activity assays and complementation analysis. The 3926 bp multisubunit fusion gene encoding the $\sim 140 \mathrm{kDa}$ DEFp polyprotein was therefore transferred to the bacterial expression vector $\mathrm{pET} 22$ downstream of the pe/B leader peptide using the EcoRI and Notl restriction sites to generate $\mathrm{pET}-\mathrm{DEFp}$.

Crude extracts of $E$. coli strain ER2566 overexpressing the DEFp polyprotein were used for enzyme activity assays, with the intact
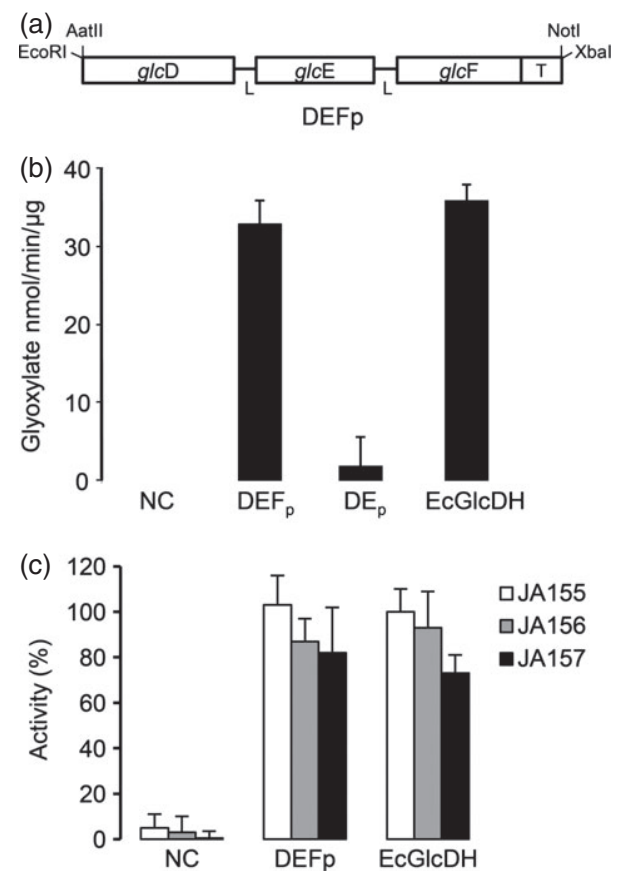

Figure 1 DEFp is functional in Escherichia coli. (a) Schematic presentation of the synthetic DEFp multisubunit fusion cassette. Abbreviations: $g / C D$, g/CE and g/cF: coding sequences for bacterial glycolate dehydrogenase subunits D, E and F; L: (Gly $\left.{ }_{4} \mathrm{Ser}\right)_{3}$ linker; $\mathrm{T}$ : His 6 polyhistidine tag. Restriction sites used for cloning of DEFp into bacterial and plant expression vectors are indicated. (b) DEFp shows glycolate dehydrogenase activity in vitro. (c) DEFp can complement the glycolate dehydrogenase activity of three $E$. coli glycolate oxidase mutants. Protein was extracted from (b) E. coli strain ER2566 and (c) the E. coli glycolate oxidase mutants JA155 (g/CD), JA156 ( $/ / C E)$ and JA157 (g/CF), and was tested for glycolate dehydrogenase activity by detecting glyoxylate formation directly. NC: overexpression of the empty $\mathrm{pET}(\mathrm{b})$ or $\operatorname{pTrc}(\mathrm{c})$ vectors. DEFp: overexpression of the $\mathrm{glcD}-\mathrm{glcE}-\mathrm{glcF}$ polyprotein. DEp: overexpression of the glcD-glcE polyprotein, EcGlcDH: overexpression of $E$. coli glycolate dehydrogenase subunits glcD-F. In (c), GlcDH activities were expressed as a percentage of the $E$. coli wild-type enzyme, which was set to $100 \%$. The data represent the mean \pm standard deviation of three biological replicates.

E. coli glycolate dehydrogenase $(E c G \mid c D H)$ and DEp polyprotein lacking the $\mathrm{F}$ subunit used as controls. As shown in Figure $1 \mathrm{~b}$, DEFp showed significant glycolate dehydrogenase activity comparable to that of the intact $E$. coli enzyme. The absence of the $F$ subunit (DEp) completely abolished enzyme activity, confirming the importance of this subunit for the maintenance of GlcDH activity.

We next investigated whether DEFp was able to complement $E$. coli glycolate oxidase mutants carrying transposon insertions in the $g / C D, g / c E$ and $g / c F$ subunits of the g/c operon. These mutants cannot grow on glycolate as a sole carbon source and do not produce detectable glycolate dehydrogenase activity (Pellicer et al., 1996). The overexpression of DEFp in all three mutant strains restored the ability of the bacteria to grow on glycolatecontaining media (data not shown). Furthermore, all three mutants yielded detectable levels of glycolate dehydrogenase activity when transformed with DEFp (Figure 1c). Taken together, these results confirmed that the engineered polyprotein retained the functionality of the native $E$. coli glycolate dehydrogenase complex. 


\section{Generation and characterization of DEFp transgenic potato plants}

To study the effects of DEFp accumulation in planta, the pTRA35S-rbcs-cTP:DEFp construct (Figure 2a) was introduced into potato plants by Agrobacterium-mediated transformation. Transformed plants were regenerated under kanamycin selection and screened for the presence of the DEFp transcript and recombinant protein. Sixty-four transgenic lines (named DEFp-1 to DEFp-64) contained the transgene and produced DEFp with the anticipated molecular size of $142 \mathrm{kDa}$ (Figure 2b). DEFp accumulated to levels between 0.05 and $0.15 \%$ of total soluble protein (TSP). Transgenic lines accumulating low ( 0.05\% TSP, DEFp-27), moderate $(\sim 0.09 \%$ TSP, DEFp-12) and high $(\sim 0.14 \%$ TSP, DEFp21) levels of recombinant DEFp were selected for vegetative propagation and were analyzed for GlcDH activity and photosynthetic performance. The recombinant protein accumulated in the vegetatively propagated plants to similar levels as the parental lines ( 0.04\% TSP, DEFp-27; 0.1\% TSP, DEFp-12; 0.15\% TSP, DEFp-21).

To investigate whether the DEFp was active in planta, GlcDH activity was measured in chloroplast extracts of 6-week-old transgenic plants accumulating different amounts of the polyprotein (lines 12, 21 and 27). Wild-type potato plants assayed under the same conditions showed significant background activity as previously described in Arabidopsis (Kebeish et al., 2007).

(a)
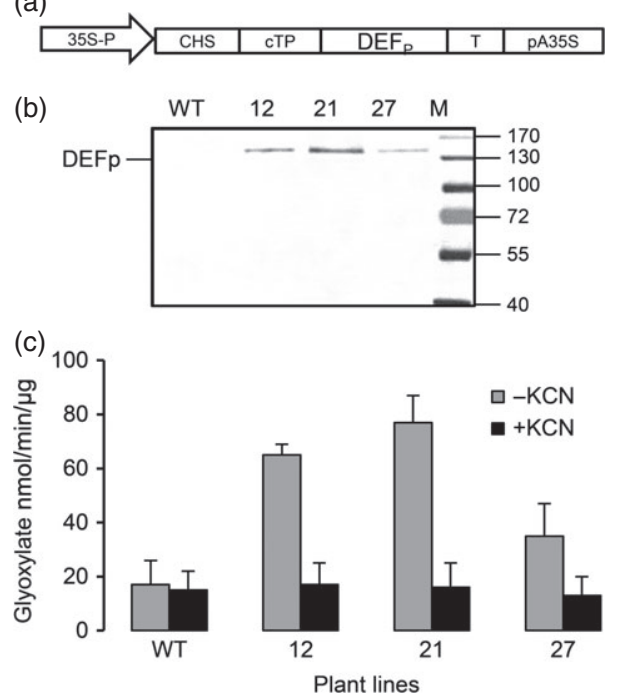

Figure 2 Generation of DEFp transgenic potato plants. (a) Plant expression cassette targeting DEFp to the chloroplast. Abbreviations: 35SP, double-enhanced CaMV 35 S promoter; CHS, 5'-untranslated region of the chalcone synthase gene; cTP: potato rbcS1 gene coding for the chloroplast targeting peptide; $\mathrm{T}$ : His 6 polyhistidine tag; pA35S: CaMV terminator sequence. (b) Immunoblot analysis of $10 \mu \mathrm{g}$ crude total soluble protein (TSP) extracted from the leaves of DEFp transgenic potato plants. The anti-His 6 antibody was used for detection of the $140 \mathrm{kDa}$ DEFp. M: prestained protein marker; 12,21 , and 27: TSP extracts from representative transgenic lines; WT: TSP extract from wild-type potato leaves. (c) DEFp is active in potato chloroplasts. The chloroplast extract was assayed for glycolate dehydrogenase activity by direct measurement of glyoxylate formation in the presence and absence of KCN. WT: wild-type potato plant $(n=3) ; 12,21,27$ : Transgenic potato lines producing DEFp $(n=3)$. The data represent means \pm standard deviation.
Chloroplast preparations from the DEFp plants produced significantly more glyoxylate from glycolate than the wild-type plants (Figure 2c), indicating that the engineered DEFp was correctly localized in the plastids and possessed GlcDH activity. The addition of cyanide inhibited GlcDH activity, confirming that the observed activity was due to the recombinant DEFp and not endogenous peroxisomal glycolate oxidase (Nelson and Tolbert, 1970). The DEFp activity correlated with the level of recombinant protein accumulation, i.e. highest in line 21 , lower in line 12 , and lowest in line 27 (correlation coefficient $R^{2}=0.8935$, data not shown). Therefore, we concluded that the novel DEFp polyprotein was targeted to the chloroplast and was functional in planta.

\section{Photosynthetic activity of DEFp transgenic potato plants}

The impact of recombinant DEFp on the photosynthetic performance of the transgenic potato plants was determined by monitoring gas-exchange parameters in the youngest fully expanded leaf. The apparent photosynthetic rate (A) in DEFp lines 12 and 21 (with the highest GlcDH activity) increased by $18 \%$ and $34 \%$, respectively, under ambient conditions (400 ppm $\mathrm{CO}_{2}, 21 \% \mathrm{O}_{2}, 24{ }^{\circ} \mathrm{C}$ ) (Table 1). The maximum $\mathrm{CO}_{2}$ fixation rate did not change significantly in any of the lines at saturating $\mathrm{CO}_{2}$ concentration, indicating that the photosynthetic rates under ambient conditions is dependent on the competition of oxygen and $\mathrm{CO}_{2}$ for binding to RuBisCO, suggesting that the increased photosynthetic rate in the transgenic lines reflects the greater availability of $\mathrm{CO}_{2}$ in the vicinity of RiBisCO. To estimate the amount of $\mathrm{CO}_{2}$ competing with $\mathrm{O}_{2}$ for RuBisCO binding in the plastids, the $\mathrm{O}_{2}$ inhibition of photosynthesis was calculated in the control plants and DEFp lines 12 and 21 (Table 1). Oxygen inhibition was significantly lower in the transgenic plants, falling $12 \%$ in line 12 and $19 \%$ in line $21 \quad(P<0.05$ and $P<0.005$, respectively) compared to the control, providing evidence for a

Table 1 Photosynthetic performance of wild-type and DEFp transgenic potato plants

\begin{tabular}{|c|c|c|c|}
\hline \multirow{2}{*}{$\begin{array}{l}\text { Photosynthesis } \\
\text { parameter }\end{array}$} & \multicolumn{3}{|l|}{ Potato lines } \\
\hline & WT & DEFp12 & DEFp21 \\
\hline $\begin{array}{l}A_{\max }\left(\mu \mathrm{mol} / \mathrm{m}^{2} / \mathrm{s}^{1}\right) \\
\left(\text { at } C_{a}=400 \text { p.p.m) }\right.\end{array}$ & $16.5 \pm 0.9$ & $19.8 \pm 0.5^{\star}$ & $22.7 \pm 1.3^{* *}$ \\
\hline $\begin{array}{l}A_{\max }\left(\mu \mathrm{mol} / \mathrm{m}^{2} / \mathrm{s}\right) \\
\left(\text { at } C_{a}=2000 \text { p.p.m) }\right.\end{array}$ & $40.6 \pm 2.7$ & $41.5 \pm 2.4$ & $40.2 \pm 1.6$ \\
\hline $\mathrm{O}_{2}$ inhibition (\%) & $34.9 \pm 4.2$ & $30.75 \pm 2.1$ ** & $28.25 \pm 1.3^{* *}$ \\
\hline$\Gamma$ (p.p.m $\mathrm{CO}_{2}$ ) & $49.6 \pm 6.3$ & $45.7 \pm 1.52^{* *}$ & $41.15 \pm 3.9^{* *}$ \\
\hline$q_{p}$ & $0.54 \pm 0.07$ & $0.55 \pm 0.06$ & $0.53 \pm 0.09$ \\
\hline$q_{N}$ & $0.77 \pm 0.01$ & $0.76 \pm 0.02$ & $0.76 \pm 0.02$ \\
\hline $\mathrm{Fv} / \mathrm{Fm}$ & $0.80 \pm 0.01$ & $0.79 \pm 0.01$ & $0.79 \pm 0.01$ \\
\hline ФPSII & $0.25 \pm 0.04$ & $0.25 \pm 0.05$ & $0.26 \pm 0.06$ \\
\hline
\end{tabular}

A: apparent $\mathrm{CO}_{2}$ assimilation; $\mathrm{C}_{\mathrm{a}}$ : $\mathrm{CO}_{2}$ concentration in the measuring cuvette; $\Gamma$, apparent $\mathrm{CO}_{2}$ compensation point; $\mathrm{F}_{\mathrm{v}} / \mathrm{F}_{\mathrm{m}}$ : maximum quantum efficiency of photosystem II; $\Phi$ PSII: efficiency of PSII photochemistry; $q_{p}$ : photochemical quenching; $q_{N}$ : nonphotochemical quenching. Vegetatively propagated plants cultivated in the phytotron or greenhouse (six plants per line) were analyzed for each measurement in two independent experiments. Values represent means \pm SD for wild-type $(\mathrm{WT})$ control and transgenic lines $(n=12)$. No significant difference in photosynthetic performance was observed between plants grown in the phytotron or greenhouse.

$* P<0.05 ; * * P<0.005 ; \pm$, standard deviation. 
higher $\mathrm{CO}_{2} / \mathrm{O}_{2}$ ratio in the vicinity of RuBisCO. Furthermore, there were no differences in the maximum quantum efficiency of photosystem II, photochemical and nonphotochemical quenching, and the Fv/Fm ratio. Plastid electron transport, membrane energization and energy dissipation therefore remained unchanged in the transgenic lines. Lines 21 and 12 were characterized by a significant $(P<0.01)$ reduction in the apparent $\mathrm{CO}_{2}$ compensation point (18\% and $9.4 \%$, respectively), indicating a higher rate of photosynthesis at low $c_{i}$ (supplementary Figure S1). These results provided additional evidence to confirm the functionality of the engineered DEFp enzyme, which was able to catalyze the conversion of glycolate to glyoxylate. The reduction in $\mathrm{CO}_{2}$ compensation point and $\mathrm{O}_{2}$ inhibition indicated that the resulting glyoxylate was oxidized further to $\mathrm{CO}_{2}$ within the plastids. Chloroplasts may oxidize glycolate completely to $\mathrm{CO}_{2}$, producing glyoxylate as an intermediate step (Frederick et al., 1973; Goyal and Tolbert, 1996; Kebeish et al., 2007). Recently, Blume et al. (2013) reported that the tobacco chloroplast pyruvate dehydrogenase complex (PDC) can decarboxylate glyoxylate and may therefore participate in the conversion of glycolate to $\mathrm{CO}_{2}$ in the plastid. The $\mathrm{CO}_{2}$ released due to the activity of the PDC complex could be directly re-fixed by RuBisCO, generating higher levels of photoassimilates. This hypothesis is supported by the higher carbohydrate content (Figure 3) and biomass production (see below) in DEFp lines. Furthermore, less ammonia would need to be re-assimilated, thus potentially improving nitrogen use efficiency. Preliminary results from our laboratory indicate that DEFp expression in chloroplast of tobacco plants improves their ability to thrive under conditions of nitrogen limitation (unpublished results).

\section{Leaf carbohydrates and other metabolites in DEFp transgenic plants}

To determine the impact of DEFp on primary carbon metabolism, we evaluated the ability of transgenic and wild-type plants to accumulate photosynthesis end products using GC-MS to measure the corresponding metabolite pool. We focused on 31 metabolites, but only those selected for physiological importance and/or significant modulation ( $t$-test $P<0.05$ ) are shown in Figure $3 a, b$. Metabolite levels relative to the standard ribitol, are presented in the supplementary Table S1. The DEFp transgenic lines showed a significant $(P<0.05)$ increase in the rapidly metabolized monosaccharaides glucose (2.1/5.8-fold in lines 12/ 21 ) and fructose (1.5/3.8-fold in lines 12/21), and the major
Figure 3 Metabolite levels in the leaves of transgenic and wild-type potato plants.

(a) Comparative carbohydrate analysis.

(b) Comparative amino and organic acid analysis. Samples were collected $5 \mathrm{~h}$ after illumination from five well-expanded leaves representing six plants per transgenic line (and four wild-type controls). The samples from each plant were pooled and analyzed by GC-MS. The ratio between mean relative response from the transgenic lines ( $12=$ white bars, $21=$ grey bars) $(n=6)$, and wild-type plants $(n=4)$ are plotted. Values $<0$ indicate lower metabolite levels in the transgenic lines compared to the control, whereas values $>0$ represent an increase. The significance of the changes was evaluated using Student's t-test. (c) Starch content of leaves of DEFp and wild-type plants. Starch content was analyzed at the beginning $(7: 00 \mathrm{am})$ and end $(21: 30 \mathrm{pm})$ of the illumination period. Data represent means \pm standard deviation from three independent pools extracted from five wellexpanded leaves of each line $(n=3)$. Statistical differences are indicated: $*=P<0.05$, ** $=P<0.005$.

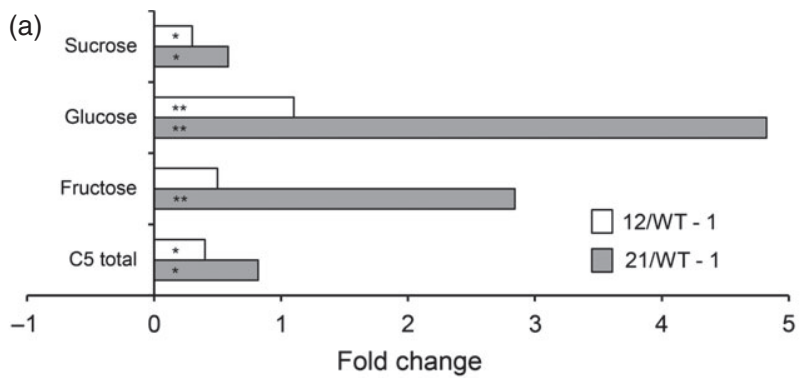

(b)

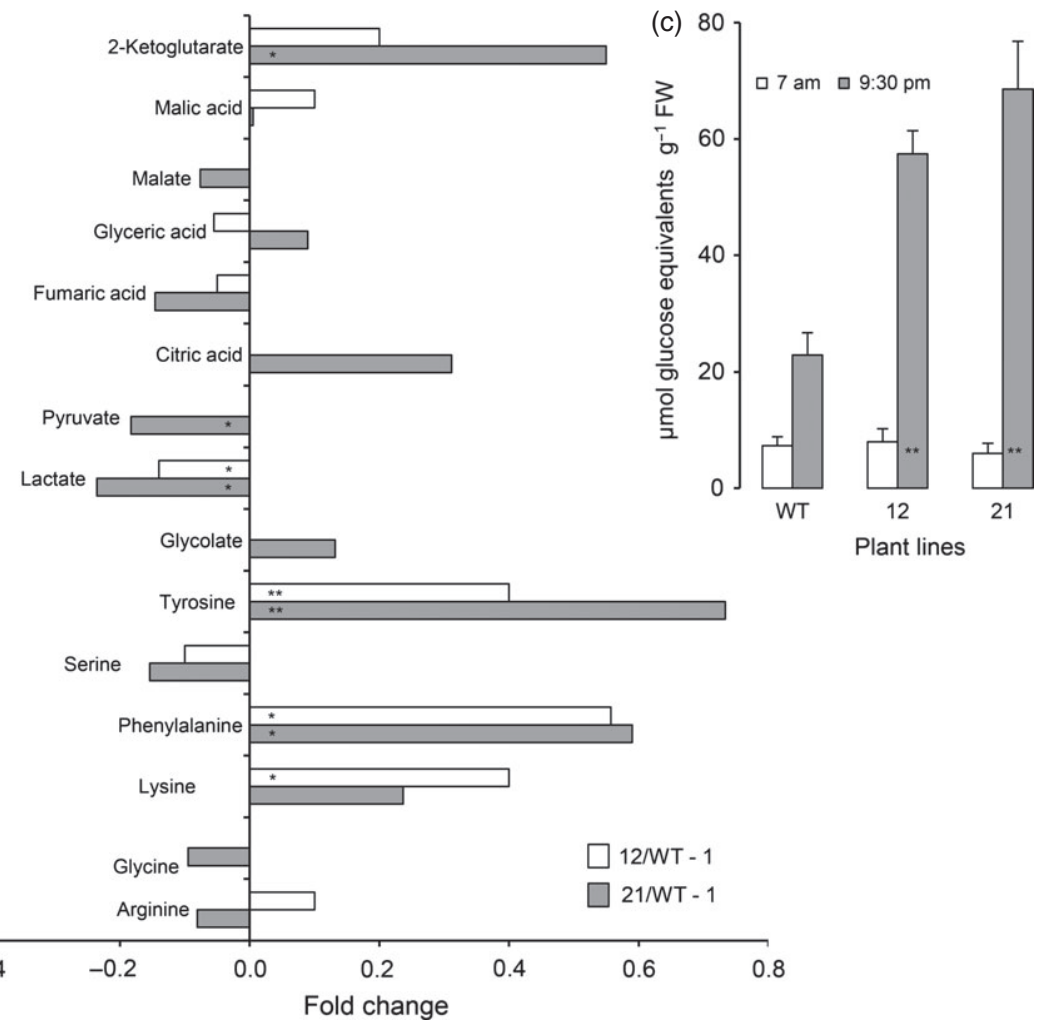


exported disaccharide sucrose (1.3/1.6-fold in lines 12/21). The most dramatic increase was observed in line 21 , with the highest DEFp levels and highest GICDH activity. In addition, the amounts of $\mathrm{C} 5$ carbohydrates in these lines were also significantly higher (1.4-fold and 1.8-fold, respectively).

Figure $3 \mathrm{~b}$ shows the relative levels of additional metabolites. Lactate levels declined in both transgenic lines, whereas pyruvate and maleate levels declined only in line 21. Both DEFp lines accumulated significantly higher amounts of 2-ketoglutarate, tyrosine and lysine than wild-type plants, but there were no significant differences in the levels of glycolate, glyceric and citric acids. The glycine to serine ratio also declined in the transgenic lines compared to controls, suggesting a reduction in the level of photorespiration metabolites by diversion to the peroxisomes and mitochondria, although the tendency was not statistically significant (data not shown).

In many plant species the immediate products of photosynthetic carbon assimilation in the light are divided between sucrose (immediately available for growth) and starch, which accumulates in the leaf through the day and is degraded to produce sucrose at night (Geiger et al., 2000; Gibon et al., 2004; Lu et al., 2005). We analyzed the content of starch (the major storage compound) in the leaves at two different time points: the beginning and end of the illumination period (Figure $3 c$ ). The leaves of all lines contained low levels of starch early in the morning but significant increases $(2.5$-fold $/ P<0.005$ in line 12 and threefold $/ P<0.005$ in line 21) were observed by the end of the day in transgenic lines compared to wild-type control. The greater increase in the transgenic lines is reflected the more efficient assimilation of $\mathrm{CO}_{2}$. Ferreira et al. (2010) showed that only $70 \%$ of the starch synthesized in potato leaves during the day is broken down during the dark phase to provide nocturnal sucrose to sink tissues, whereas $100 \%$ is broken down in Arabidopsis (Gibon et al., 2004; Schneider et al., 2002). However, the results presented in Figure $3 c$ show almost complete starch degradation during the night in the best-performing DEFp lines, indicating that the transitory starch is either converted to sucrose and exported from the leaf to the sink organs, or used for dark respiration and biomass accumulation in the leaf (Graf and Smith, 2011).

The increase in starch content did not occur at the expense of other carbohydrates, because DEFp lines 12 and 21 (with the highest GlcDH activity and photosynthesis rates) also produced higher levels of glucose, fructose and sucrose (Figure 3a). Photosynthetic carbon assimilation was sufficient to support not only growth, but also the accumulation of storage compounds in the leaf, which are then mobilized to provide carbon for growth during the night.

\section{Phenotypic effects of DEFp expression}

We also investigated whether the enhanced photosynthetic performance of the transgenic plants resulted in phenotypic differences and higher biomass accumulation. Indeed, tubers harvested from lines 21 and 12 with the highest levels of GlcDH activity, and the most significant reduction in $\mathrm{CO}_{2}$ compensation point and $\mathrm{O}_{2}$ inhibition, showed accelerated sprouting (1 week earlier than normal) and developed $25 \%$ more $(P<0.05 ; n=10)$ shoots per plant than control lines. The transgenic lines also produced more $(9 \pm 2 ; P<0.05 ; n=10)$ leaves and broader stems than the control plants (Figure 4a). Studies on the manipulation of photosynthetic carbon fixation suggest that plants evolved mechanisms to ensure co-ordination of leaf development and metabolism (reviewed by Raines and Paul, 2006). Our results support the idea of Raines and Paul (2006) that changes in photosynthetic carbon assimilation, resulting in increased availability of carbohydrates not only have an impact on plant yield, but also play a role in modulating the developmental programme of the plant.

Although the mechanisms governing release from dormancy and the initiation of sprouting are not completely understood (Suttle, 1996), starch degradation and sucrose biosynthesis are tightly linked to tuber sprouting (Farré et al., 2001). Furthermore, sucrose levels are responsible for the regulation of metabolic (a) WT
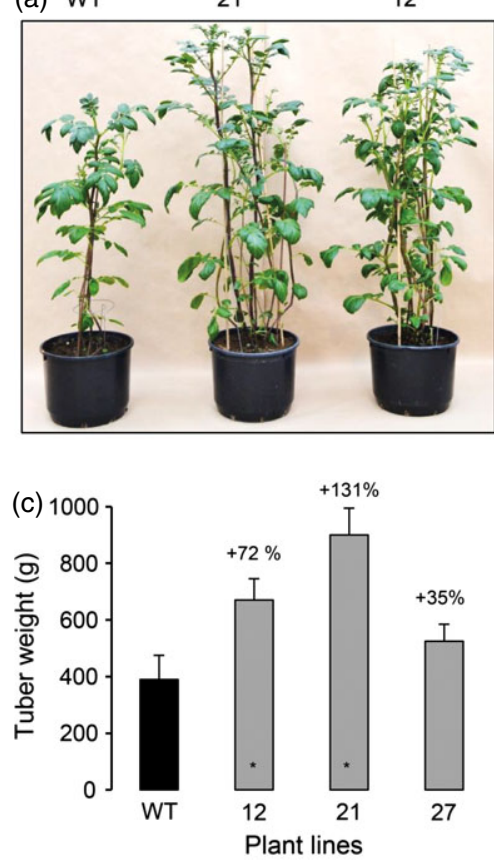

(b) WT

21

12

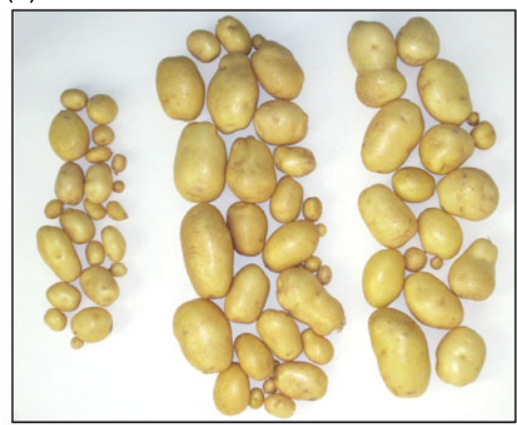

(d)

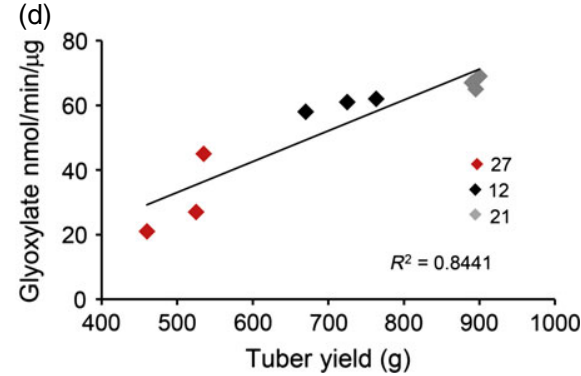

Figure 4 Impact of DEFp expression on potato phenotype and tuber yield. (a) Phenotypic appearance of 8-week-old representative wildtype plant and DEFp lines 21 and 12, planted in soil 6 months after harvest. (b) The phenotype of tubers from one representative wild-type plant, and transgenic lines 12 and 21, 2 days after harvest. (c) Tuber yield per plant in wild-type and DEFp transgenic lines 12, 21 and 27. Data represent means \pm standard deviations $(n=15)$. $* P<0.005$. The increase in tuber yield of the DEFp lines is indicated as a percentage compared to wild-type tubers. (d) Relationship between $\mathrm{GlCDH}$ activity (in $\mathrm{nmol} / \mathrm{min} / \mu \mathrm{g}$ ) and tuber yield of transgenic lines 12,21 and 27. $R^{2}$ : correlation coefficient. The GlcDH activity of the wild-type control was subtracted from the activity measured in the DEFp lines. 
processes during the sink to source transition in potato tubers (Hajirezaei et al., 2003). The metabolic analysis of DEFp-21 tubers showed they contained 1.2-fold higher $(P<0.05)$ levels of sucrose than wild-type tubers, which would affect their sprouting behaviour.

The constitutive production of DEFp led to a significant increase in tuber yield in the transgenic lines (Figure $4 b, c$ ), 2.3-fold/ $P<0.005$ in line 21 , followed by 1.7 -fold $/ P<0.05$ in line 12 and 1.3-fold (not statistically significant but nevertheless a trend in line $27(p<0.1)$. The tuber yield in the transgenic lines correlated strongly with $\mathrm{GlCDH}$ activity $\left(R^{2}=0.8441\right.$; Figure $\left.4 \mathrm{~d}\right)$. These results were consistent in three different experiments carried out over a period of 2 years.

Similar phenotypic effects were observed in transgenic Arabidopsis plants expressing all three subunits of the bacterial glycolate dehydrogenase (Kebeish et al., 2007). The transgenic plants grew faster, produced more shoot and root biomass and contained more soluble sugars, reflecting the increased $\mathrm{CO}_{2}$ concentration in the vicinity of RuBisCO. Furthermore, the introduction of a complete glycolate catabolic cycle into Arabidopsis chloroplasts reduced the photorespiratory flux, increased the photosynthesis rates and improved plant growth (Maier et al., 2012).

The strong correlation between GlcDH activity, photosynthesis rates and tuber yield suggest that the significant increase in photosynthesis rates and the accumulation of end-product metabolites was able to enhance the sink capacity of DEFp transgenic plants. The higher tuber yield did not occur at the expense of leaf starch and primary metabolites (Figure 3). Sucrose is the major form in which fixed carbon is transported to the sink organs. Thus, the rate of sucrose synthesis is likely to be important for sink development and final crop yield, demonstrating the central role of sugars in the coordination of carbon supply and plant growth as previously described by Smith and Stitt (2007). There was no impact on photosynthetic parameters and tuber yield when photosynthetic sucrose biosynthesis was reduced by suppressing cytosolic fructose-1,6-bisphosphate in potato plants (Zrenner et al., 1996). However, our study demonstrated that a 1.7-fold increase in GlcDH activity led to a significant increase in photosynthesis, boosting the accumulation of assimilates, and ultimately biomass and tuber yield. The yield differences reported here are much higher than expected, but transgenic potatoes modified to simultaneously enhance sugar export form the leaf and sugar uptake in the tuber also had a higher starch content and tuber yield (Jonik et al., 2012). This indicates that substantial changes in yield can be achieved by the targeted manipulation of metabolic pathways.

\section{Conclusion}

We have established a powerful approach to increase the biomass of potato plants by improving photosynthetic carbon fixation using a glycolate dehydrogenase polyprotein. The constitutive expression of DEFp in potato chloroplasts boosted photosynthetic efficiency and carbohydrate metabolism. Changes in the photosynthetic capacity of the plants were directly reflected in the phenotype, i.e. more leaves, a thicker stem and a 2.3-fold increase in tuber yield. Molecular and biochemical analysis revealed a strong correlation between GlcDH activity, the photosynthetic performance and overall yield. This is the first study describing such a substantial photorespiratory bypass effect in a crop species. As previously suggested, it may be possible to improve the yield further by boosting the sink strength (Jonik et al., 2012; Zhang et al., 2008). Further work is required to understand the mode of action of DEFp, the extent of the metabolic changes, and the feasibility of this strategy to improve yields in other crop species.

\section{Experimental procedures}

\section{Engineering the recombinant GlcDH polyprotein}

The multi-subunit fusion cassette DEFp contained the E. coli genes $g / c D, g / c E$ and $g / c F$ in tandem ( $E$. coli K12 genome sequence: gi49175990) and was codon optimized for Brassica napus and synthesized by Entelechon $\mathrm{GmbH}$ (Bad Abbach, Germany). A genetic algorithm was also used to optimize the synthetic genes simultaneously for a large set of competing parameters, such as mRNA secondary structure, cryptic splice sites, codon and motif repeats, and homogenous GC content. The synthesized DEFp-CDNA was transferred to the EcoRI and Xbal restriction sites of pUC18 to generate plasmid pUC-DEFp.

\section{Plasmid DNA, bacteria and plants}

The plasmids pET22b(+) (Novagen, Darmstadt, Germany) and pTrc99a (Pharmacia, Freiburg, Germany) were used for protein expression in bacteria, and pTRA was used in plants (Sack et al., 2007). Escherichia coli strain DH5 $\alpha$ was used for general cloning and ER2566 (New England Biolabs, Frankfurt, Germany) was used for protein expression. Strains JA155 (araD $\Delta / a c$ rpsL flbB deoC ptsF rbsR glcD::cat), JA156 (araD $\Delta$ lac rpsL flbB deoC ptsF rbsR glcE::cat) and JA157 (araD $\Delta / a c$ rpsL flbB deoC ptsF rbsR glcF::cat) were used for complementation experiments (Pellicer et al., 1996). Agrobacterium tumefaciens strain GV3101 (pMP90RK, $\mathrm{Gm}^{R}, \mathrm{Km}^{\mathrm{R}}, \mathrm{Rif}^{\mathrm{R}}$ ) was used for plant transformation (Konz and Schell, 1986). Leaf discs from 4-5-week-old wild-type potato plants (Solanum tuberosum CV. Bintje) were transformed by infection with Agrobacterium tumefaciens carrying binary vector pTRA-DEFp (Dietze et al., 1995). Potato plants were cultivated in the phytotron and greenhouse in DE73 standard soil in 13-L pots with a 16 -h natural daylight photoperiod and $21 / 18{ }^{\circ} \mathrm{C}$ day/night temperature. In phytotrons, metal-halide lamps at light intensity of $100-110 \mu \mathrm{E}$ were used. Unless otherwise specified, the data presented here are average of greenhouse conditions. Tubers were harvested when the plants entered senescence. Plants were propagated by cuttings and vegetative multiplication of tubers. Tubers of the same weight $(\sim 30 \mathrm{~g})$ were chosen for plant propagation.

\section{Construction of the bacterial and plant expression cassettes}

$\mathrm{DEF}_{\mathrm{p}}$ was subcloned from pUC18-DEFp into the bacterial expression vector $\mathrm{pET} 22 \mathrm{~b}(+)$ downstream of the pe/B leader peptide using the EcoRI and Notl restriction sites, generating vector $p E T$ DEFp. This was facilitated by shuttling the DEFp cassette into pTRA upstream of the Cauliflower mosaic virus (CaMV) 35S terminator sequence at the EcoRI/Xbal restriction sites. The constitutive CaMV 35 S promoter (Kay et al., 1987), the $5^{\prime}$-untranslated region of the chalcone synthase gene and the chloroplast targeting peptide from the potato rbcS1 gene (gi21562) were amplified by PCR using vector pTRAkc-rbcs1-cTP as the template (Kebeish et al., 2007). The amplified 35S-rbcs-cTP PCR product was subcloned into the shuttle vector using Ascl/ Aatll restriction sites introduced by $P C R$, yielding the final plant expression vector pTRA-35S-rbcs-cTP: DEFp. 


\section{Protein expression and complementation analysis}

Escherichia coli ER2566 cells (carrying pET-DEFp, pER-DEp, pET$\mathrm{EcGl} C \mathrm{CH}$ or empty $\mathrm{pET}$ ) were cultivated in $200 \mathrm{~mL} \mathrm{LB}$ medium until the $\mathrm{OD}_{600}$ reached 0.5 . The $\mathrm{pET}-\mathrm{EcGl} \mathrm{cDH}$ vector contained part of the E. coli g/c operon encoding the separate glycolate dehydrogenase $D, E$ and $F$ subunits (Bari et al., 2004). Expression was induced by adding $\beta$-D-isopropyl-thiogalactopyranoside (IPTG) to a final concentration of $1 \mathrm{~mm}$ and incubating for $2 \mathrm{~h}$ at $37^{\circ} \mathrm{C}$. The cells were then washed in $10 \mathrm{~mm}$ potassium phosphate $(\mathrm{pH} 8.0)$ and resuspended in $1 \mathrm{~mL}$ of the same buffer. The cells were lysed by sonication on ice ( 4 cycles, $4 \times 30$ s; Sonicator Bandelin Sonopulus GM 70, Berlin Germany) and centrifuged at $30000 \mathbf{g}$ for $25 \mathrm{~min}$ at $4{ }^{\circ} \mathrm{C}$.

Escherichia coli mutants JA155, JA156 and JA157 deficient for g/CD, g/CE and g/CF subunits of endogenous glycolate dehydrogenase, respectively (Pellicer et al., 1996), were transformed with the vectors pTrc99a-DEFp and pTrc-EcGlcDH (Bari et al., 2004) plasmids and cultivated for 2 days in minimal medium (Miller, 1972) using glycolate as the sole carbon source, supplemented with appropriate antibiotics $(25 \mu \mathrm{g} / \mathrm{mL}$ chloramphenicol and $100 \mu \mathrm{g} / \mathrm{mL}$ ampicillin). The cells were diluted into $3 \mathrm{~L}$ of fresh medium and grown to an $\mathrm{OD}_{600}$ of $0.7-0.9$. Protein expression was induced by adding $0.5 \mathrm{~mm}$ IPTG and the cells were incubated for a further $3 \mathrm{~h}$ at $37{ }^{\circ} \mathrm{C}$ followed by the extraction of soluble proteins for the glycolate dehydrogenase assay as described below. The concentration of extracted soluble protein was determined in triplicate by the Bradford assay against bovine serum albumin (BSA) standards.

\section{Total protein extraction and immunoblot analysis}

The upper fully expanded leaves from 6-week-old potato plants were ground to a fine powder under liquid nitrogen, and total soluble protein (TSP) was extracted as described by Nölke et al. (2008) with two volumes of extraction buffer [50 mM Tris- $\mathrm{HCl}, \mathrm{pH}$ 8, $100 \mathrm{~mm} \mathrm{NaCl}, 10 \mathrm{~mm}$ dithiothreitol (DTT), $5 \mathrm{~mm}$ ethylenediaminetetraacetic acid (EDTA) and $0.1 \%(\mathrm{v} / \mathrm{v})$ Tween-20]. The extracts were centrifuged at $8500 \mathrm{~g}$ for $20 \mathrm{~min}$ at $4{ }^{\circ} \mathrm{C}$ and used for immunoblot analysis. DEFp was detected with a rabbit anti$\mathrm{His}_{6}$ monoclonal antibody (RAb-His; $200 \mathrm{ng} / \mathrm{mL}$ ) and a horseradish peroxidase-conjugated goat-anti-rabbit secondary antibody (GAR ${ }^{A P} ; 120 \mathrm{ng} / \mathrm{mL}$ ) (Jackson ImmunoResearch Laboratories, Suffolk, UK). DEFp band intensities were quantified using Aida software (Raytest, Straubenhardt, Germany) against known concentrations of bacterial affinity-purified DEFp as a standard.

\section{Isolation of chloroplasts from potato leaves}

Intact chloroplasts were isolated from 6-week-old potato plants as described by Goyal et al. (1988). Leaf material (5 g) was ground in $100 \mathrm{~mL}$ grinding buffer (50 mM HEPES-KOH pH 7.5, $1 \mathrm{~mm}$ $\mathrm{MgCl}_{2}, 1 \mathrm{~mm}$ EDTA, $1 \mathrm{~g} / \mathrm{L}$ BSA, $0.2 \mathrm{~g} / \mathrm{L}$ sodium ascorbate, $0.3 \mathrm{~m}$ mannitol, $5 \mathrm{~g} / \mathrm{L}$ polyvinylpyrrolidone) and all subsequent steps were performed in the dark at $0{ }^{\circ} \mathrm{C}$. Crude protein extract was filtrated through three layers of Miracloth and the solution was centrifuged at $1000 \mathrm{~g}$ for $10 \mathrm{~min}$ at $0^{\circ} \mathrm{C}$. The pellets were resuspended in $1 \mathrm{~mL}$ SH-buffer (50 mM HEPES-KOH $\mathrm{pH} 7.5$, $0.33 \mathrm{M}$ sorbitol) and $1 \mathrm{~mL}$ of the solution was loaded onto $8-\mathrm{mL}$ $35 \%$ (v/v) Percoll gradient (35\% Percoll, 65\% SH-buffer). The gradient was centrifuged at $500 \mathrm{~g}$ for $5 \mathrm{~min}$ at $0^{\circ} \mathrm{C}$. The chloroplast pellet was washed in $1 \mathrm{~mL} \mathrm{SH}$-buffer and chloroplast proteins were extracted in $500 \mu \mathrm{L}$ extraction buffer $(50 \mathrm{~mm}$ HEPES- $\mathrm{NaOH}$ pH 7.5, 2 mm EDTA, 5 mm MgCl $2,0.1 \%$ (v/v) Triton
X-100, 20\% (v/v) glycerol). The purity (> 95\%) of the chloroplast fraction was confirmed by catalase and fumarase activity assays (Figure S2) as previously described (Ferri et al., 1978). The chloroplast proteins were directly used in the glycolate dehydrogenase activity assay.

\section{Glycolate dehydrogenase assay}

Glycolate dehydrogenase activity was determined as described by Lord (1972) using $40 \mu \mathrm{g}$ of bacterial crude protein extract or chloroplast proteins added to $150 \mu \mathrm{L}$ of buffer $(10 \mathrm{~mm}$ potassium phosphate, $\mathrm{pH} 8.0,0.025 \mathrm{~mm}$ phenazine methosulfate, $10 \mathrm{~mm}$ potassium glycolate). At fixed time points (1, 2, 3, 4, 5 and $10 \mathrm{~min}$ ) individual assays were terminated by adding $30 \mu \mathrm{L} 12 \mathrm{~m}$ $\mathrm{HCl}$. After incubation for $10 \mathrm{~min}, 70 \mu \mathrm{L} 0.1 \mathrm{~m}$ phenylhydrazine was added and the mixture was again incubated at room temperature for $10 \mathrm{~min}$. The extinction due to the formation of glyoxylate phenylhydrazone was measured at $324 \mathrm{~nm}$ in a quartz 96-well microtiter plate using a Synergy HT-I multiplate reader (BioTec, Bad Friedrichshall, Germany).

\section{Gas-exchange measurements}

Fully expanded upper leaves from 7-week-old potato plants were used for gas-exchange measurements in LI-6400 system (Li-Cor), as previously described (Kebeish et al., 2007). The following parameters were used: photon flux density $1000 \mathrm{mmol} / \mathrm{m}^{2} / \mathrm{s}$, chamber temperature $26{ }^{\circ} \mathrm{C}$, flow rate $150 \mathrm{mmol} / \mathrm{s}$, relative humidity $60-70 \%$. The oxygen inhibition of carbon assimilation (A) was calculated from $A$ at $C_{a}=400 \mathrm{ppm}$, and atmospheric oxygen concentrations of $21 \%$ and $2 \%$, using the following equation:

$$
\text { oxygen-inhibition }(\%)=\left(1-A_{21} / A_{2}\right) \times 100 \text {. }
$$

The $\mathrm{CO}_{2}$ compensation point $(\Gamma)$ was determined by measuring the photosynthesis rates at 400,300,200, 100, 80, 60 and $40 \mathrm{ppm} \mathrm{CO}_{2}$. The apparent $\mathrm{CO}_{2}$ compensation point $(\Gamma)$ was deduced from $\mathrm{A} / \mathrm{Ci}$ curves by regression analysis in the linear range of the curve. Measurements were taken from the same plants after $4 \mathrm{~h}$ light on two different days. After these measurements, leaf samples were used to determine glycolate dehydrogenase activity and carbohydrate levels.

\section{Sugar and starch quantities in potato leaves}

For sugar analysis, $40 \mathrm{mg}$ of leaf material harvested from five well-expanded leaves from 7-week-old plants after $4 \mathrm{~h}$ of illumination was flash frozen in liquid nitrogen and ground in $1 \mathrm{~mL}$ prechilled chloroform/methanol/water (2.5/1/1 v/v/v). The extract was incubated at $4{ }^{\circ} \mathrm{C}$ for $10 \mathrm{~min}$ with moderate shaking and centrifuged at $16000 \mathrm{~g}$ for $2 \mathrm{~min}$ at $4{ }^{\circ} \mathrm{C}$ before $500 \mu \mathrm{L}$ of the supernatant was mixed with $250 \mu \mathrm{L}$ of water. The samples were centrifuged as above, the top layer was collected and dried in a speed vacuum concentrator and the glucose, fructose and sucrose concentrations were determined enzymatically (Stitt et al., 1989). For starch measurements, $50 \mathrm{mg}$ leaf material was collected in two different time points, at the beginning (7:00 am, after $1.5 \mathrm{~h}$ illumination) and at the end of the illumination period (21:30). The frozen leaf material was ground in liquid nitrogen and resuspended in $80 \%(\mathrm{v} / \mathrm{v})$ ethanol. The extract was mixed for $10 \mathrm{~min}$ at $80{ }^{\circ} \mathrm{C}$ and centrifuged for $20 \mathrm{~min}$. The pellet was resuspended in $80 \%(\mathrm{v} / \mathrm{v})$ and $50 \%(\mathrm{v} / \mathrm{v})$ ethanol, respectively, followed by mixing at $80{ }^{\circ} \mathrm{C}$ and centrifugation as above. The resulting pellet was washed with $90 \%(\mathrm{v} / \mathrm{v})$ ethanol, resuspended in $400 \mu \mathrm{L} 0.2 \mathrm{KOH}$ and incubated at $95{ }^{\circ} \mathrm{C}$ 
for $1 \mathrm{~h}$. Finally, samples were mixed with $70 \mu \mathrm{L} 1 \mathrm{~m}$ acetic acid and the starch content was measured enzymatically.

\section{Analysis of metabolites from potato leaves}

For metabolite analysis, samples were collected $5 \mathrm{~h}$ after illumination from five well-expanded leaves representing six plants per transgenic line and four wild-type controls. The samples from each plant were pooled and the leaf material was homogenized in liquid nitrogen. Metabolites were quantified in $20 \mathrm{mg}$ of homogenized material by GC-MS as described by Lisec et al. (2006). Chromatograms were analyzed using CHROMA TOF software (Leco Corporation, Mönchengladbach, Germany) and TAGFINDER (Luedemann et al., 2008).

\section{Statistical analysis}

Significance was determined according to Student's t-test using Excel software (Microsoft). Two-sided tests were performed for homoscedastic matrices.

\section{Acknowledgements}

The authors gratefully acknowledge Dr. Flora Schuster for producing the transgenic potato plants, Holger Spiegel for helpful discussion for construct design, Birgit Lippmann for help with GC/ MS analysis, and Dr. Richard M Twyman for critical reading of the manuscript. This work was supported by the BMBF-GABI Improve FKZ 0315038C.

\section{References}

Bari, R., Kebeish, R., Kalamajka, R., Rademacher, T. and Peterhänsel, C. (2004) A glycolate dehydrogenase in the mitochondria of Arabidopsis thaliana. J. Exp. Bot. 55, 623-630.

Bloom, A.J., Burger, M., Asensio, J.S.R. and Cousins, A.B. (2010) Carbon dioxide enrichment inhibits nitrate assimilation in wheat and Arabidopsis. Science, 328, 899-903.

Blume, C., Behrens, C., Eubel, H., Braun, H.-P. and Peterhansel, C. (2013) A possible role for the chloroplast pyruvate dehydrogenase complex in plant glycolate and glyoxylate metabolism. Phytochemistry, 95, 168-176.

Campbell, W.J. and Ogren, W.L. (1990) Glyoxylate inhibition of ribulosebiphosphate carboxylase-oxygenase: activation in intact, lysed and reconstituted chloroplasts. Photosynth. Res. 23, 257-268.

Dietze, J., Blau, A. and Willmitzer, L. (1995) Agrobacterium-mediated transformation of potato (Solanum tuberosum). In: Gene Transfer to Plants (Potrykus, I. and Spangenberg, G., eds), pp. 24-29. Berlin: Springer-Verlag.

Fahnenstich, H., Scarpeci, T.E., Valle, E.M., Flügge, U.-I. and Maurino, V.G. (2008) Generation of hydrogen peroxide in chloroplasts of Arabidopsis overexpressing glycolate oxidase as an inducible system to study oxidative stress. Plant Physiol. 148, 719-729.

FAO (2009) Coping with a changing climate: considerations for adaptation and mitigation in agriculture. In Environment and Natural Resources Service Series No. 15 (Glantz, M.H., Gommes, R., Ramasamy, S. and FAO, eds), pp. 1-20. Rome: FAO.

FAOSTAT (2012) Crop processed. Potato production in metric tonnes. GeoHive, Available at: http://www.geohive.com/charts/ag_potato.aspx

Farré, E.M., Bachmann, A., Willmitzer, L. and Trethewey, R.N. (2001) Acceleration of potato tuber sprouting by the expression of bacterial pyrophosphatase. Nat. Biotechnol. 19, 268-272.

Ferreira, S., Senning, M., Sonnewald, S., Keßling, P.-M., Goldstein, R. and Sonnewald, U. (2010) Comparative transcriptome analysis coupled to X-ray CT reveals sucrose supply and growth velocity as major determinants of potato tuber starch synthesis. BMC Genomics, 11, 1471-2164.

Ferri, G., Comerio, G., Ladarola, P., Zaponi, M.C. and Speranza, M.L. (1978) Subunit structure and activity of glyceraldehyde-3-phosphate dehydrogenase from spinach chloroplast. Biochim. Biophys. Acta, 522, 19-31.
Frederick, S.E., Gruber, P.J. and Tolbert, N.E. (1973) The occurrence of glycolate dehydrogenase and glycolate oxidase in green parts: an evolutionary survey. Plant Physiol. 52, 318-323.

Geiger, D.R., Geigenberger, P. and Stitt, M. (2000) Role of starch in carbon translocation and partitioning at the plant level. Aust. J. Plant Physiol. 27, $571-582$.

Gibon, Y., Bläsing, O.E., Palacios-Rojas, N., Pankovic, D., Hendriks, J.H., Fisahn, J., Höhne, M., Günther, M. and Stitt, M. (2004) Adjustment of diurnal starch turnover to short days: depletion of sugar during the night may leads to a temporary inhibition of carbohydrate utilization, accumulation of sugar and post-tranlational activation of ADP-glucose pyrophosphorylase in the following light period. Plant J. 39, 847-862.

Givan, C.V. and Kleczkowski, L.A. (1992) The enzymic reduction of glyoxylate and hydroxypyruvate in leaves of higher plants. Plant Physiol. 100, 552-556. Goyal, A. and Tolbert, N.E. (1996) Association of glycolate oxidation with photosynthetic electron transport in plant and algal chloroplasts. Proc. Natl Acad. Sci. USA, 93, 3319-3324.

Goyal, A., Betsche, T. and Tolbert, N.E. (1988) Isolation of intact chloroplasts from Dunaliella tertiolecta. Plant Physiol. 88, 543-546.

Graf, A. and Smith, A.M. (2011) Starch and the clock: the dark side of plant productivity. Trends Plant Sci. 16, 169-175

Hajirezaei, M.R., Börnke, F., Peisker, M., Takahata, Y., Lerchl, J., Kirakosyan, A. and Sonnewald, U. (2003) Decreased sucrose content triggers starch breakdown and respiration in stored potato tubers (Solanum tuberosum). J. Exp. Bot. 54, 477-488.

Jonik, C., Sonnewald, U., Hajirezaei, M., Flüge, U.-I. and Ludewig, F. (2012) Simultenous boosting of source and sink capacities doubles tuber starch yield of potato plants. Plant Biotechnol. J., 10, 1088-1098.

Kay, R., Chan, A. and McPherson, J. (1987) Duplication of CaMV 35 S promoter sequences creates a strong enhancer for plant genes. Science, 236, 12991302

Kebeish, R., Niessen, M., Thiruveedhi, K., Bari, R., Hirsch, H.-J., Rosenkranz, R., Stäbler, N., Schönfeld, B., Kreuzaler, F. and Peterhansel, C. (2007) Chloroplast photorespiratory bypass increases photosynthesis and biomass production in Arabidopsis thaliana. Nat. Biotechnol. 25, 593-599.

Konz, C.B. and Schell, J. (1986) The promoter of TL-DNA gene 5 controls the tissue-specific expression of chimaeric genes carried by a novel type of Agrobacterium binary vector. Mol. Gen. Genet. 204, 382-396.

Kozani, A. and Takeba, G. (1996) Photorespiration protects C3 plants from photooxidation. Nature, 384, 557-560.

Laing, W.A., Ogren, W.L. and Hageman, R.H. (1974) Regulation of soybean net photosynthetic $\mathrm{CO}_{2}$ fixation by the interaction of $\mathrm{CO}_{2}, \mathrm{O}_{2}$ and ribulose 1,5-diphosphate carboxylase. Plant Physiol. 54, 678-685.

Leegood, R.C., Lea, P.J., Adcock, M.D. and Häusler, R.E. (1995) The regulation and control of photorespiration. J. Exp. Bot. 46, 1397-1414.

Lefebvre, S., Lawson, T., Zakhleniuk, O.V., Lloyd, J.C., Raines, C.A. and Fryer, M. (2005) Increased sedoheptulose-1,7-bisphosphatase activity in transgenic tobacco plants stimulates photosynthesis and growth from an early stage in development. Plant Physiol. 138, 451-460.

Lisec, J., Schauer, N., Kopka, J., Willmitzer, L. and Fernie, A.R. (2006) Gas chromatography mass spectrometry-based metabolite profiling in plants. Nat. Protoc. 1, 387-396.

Long, S.P., Zhu, X.-G., Naidu, S.L. and Ort, D.R. (2006) Can improvement in photosynthesis increase crop yields? Plant, Cell Environ. 29, 315-330.

Lord, J.M. (1972) Glycolate oxidoreductase in Escherichia coli. Biochim. Biophys. Acta, 267, 227-237.

Lu, Y., Gehan, J.P. and Sharkey, T.D. (2005) Daylength and circadian effects on starch degradation and maltose metabolism. Plant Physiol. 138, 2280-2291.

Luedemann, A., Strassburg, K., Erban, A. and Kopka, J. (2008) TagFinder for the quantitative analysis of gas chromatography and mass spectrometry (GC-MS)-based metabolite profiling experiments. Bioinformatics, 24, 732-737.

Maier, A., Fahnenstich, H., Von Caemmerer, S., Engqvist, M.K., Weber, A.P.M., Flugge, U.-I. and Maurino, V.G. (2012) Glycolate oxidation in A. thaliana chloroplasts improves biomass production. Front. Plant Sci. 3, 38

Maurino, V.G. and Peterhansel, C. (2010) Photorespiration: current status and approaches for metabolic engineering. Curr. Opin. Plant Biol. 13, 249-256. 
Miller, J.H. (1972) Experiments in Molecular Genetics. New York: Cold Spring Harbour Laboratory Press.

Miyagawa, Y., Tamoi, M. and Shigeoka, S. (2001) Overexpression of cyanobacterial fructose-1,6-/sedoheptulose-1,7-bisphosphatase in tobacco enhances photosynthesis and growth. Nat. Biotechnol. 19, 965-969.

Nelson, E.B. and Tolbert, N.E. (1970) Glycolate dehydrogenase in green algae. Arch. Biochem. Biophys. 141, 102-110.

Nölke, G., Cobanov, P., Uhde-Holzem, K., Reustle, G., Fischer, R. and Schillberg, S. (2008) Grapevine fanleaf virus (GFLV)-specific andibodies confer GFLV and Arabis mosaic virus (ArMV) resistance in Nicotiana benthamiana. Mol. Plant Pathol. 9, 41-49.

OECD/FAO (2011) OECD-FAO Agricultural Outlook 2011-2020. OECD Publishing and FAO, Available at: http://dx.doi.org/10.1787//agr_outlook-2011-en

Pellicer, M.T., Badia, J., Aguilar, J. and Baldoma, L. (1996) glc locus of Escherichia coli: characterization of genes encoding the subunits of glycolate oxidase and the glc regulator protein. J. Bacteriol. 178, 2051-2059.

Peterhansel, C. and Maurino, V.G. (2011) Photorespiration redesigned. Plant Physiol. 155, 49-55.

Peterhansel, C., Niessen, M. and Kebeish, R.M. (2008) Metabolic engineering towards the enhancement of photosynthesis. Photochem. Photobiol. 84, 1317-1323.

Peterhansel, C., Blume, C. and Offermann, S. (2012) Photorespiratory bypasses: how can they work? J. Exp. Bot. 64, 709-715.

Rachmilevitch, S., Cousins, A.B. and Bloom, A.J. (2004) Nitrate assimilation in plant shoots depends on photorespiration. Proc. Natl Acad. Sci. USA, 101, 11506-11510.

Raines, C.A. and Paul, M.J. (2006) Products of leaf primary carbon metabolism modulate the developmental programme determining plant morphology. J. Exp. Bot. 57, 1857-1862.

Sack, M., Paetz, A., Kunert, R., Bomble, M., Hesse, F., Stiegler, G., Fischer, R., Katinger, H., Stoeger, E. and Rademacher, T. (2007) Functional analysis of the broadly neutralizing human anti-HIV-1 antibody 2F5 produced in transgenic BY-2 suspension cultures. FASEB J. 21, 1655-1664.

Schneider, A., Häusler, R.E., Kolukisaoglu, U., Kunze, R., van der Graaff, E., Schwacke, R., Catoni, E., Desimone, M. and Flügge, U.I. (2002) An Arabidopsis thaliana knock-out mutant of the chloroplast triose phosphate/ phosphate translocator is severely compromised only when starch synthesis, but not starch mobilization is abolished. Plant J. 32, 685-699.

Smith, A.M. and Stitt, M. (2007) Coordination of carbon supply and plant growth. Plant, Cell Environ. 30, 1126-1149.
Somerville, C.R. (1984) The analysis of photosynthetic carbon dioxide fixation and photorespiration by mutant selection. Oxford Surveys Plant Mol. Cell Biol. 1, 103-131.

Somerville, C.R. (2001) An early Arabidopsis demonstration. Resolving a few issues concerning photorespiration. Plant Physiol. 125, 20-24.

Stitt, M., Lilley, R., Gerhard, R. and Heldt, H. (1989) Determination of metabolite levels in specific cells and subcellular compartments of plant leaves. Methods Enzymol. 174, 518-552.

Suttle, J.C. (1996) Dormancy in tuberous organs: problems and perspectives. In Plant Dormancy, Physiology, Biochemistry and Molecular Biology. (Lang, G.A., ed.), pp. 133-143. Oxon, UK: Lab International.

Tolbert, N.E. (1997) The C2 oxidative photosynthetic carbon cycle. Annu. Rev. Plant Physiol. Plant Mol. Biol. 48, 1-25.

Wang, F. (2008) The importance of quality potato seed in increasing potato production in Asia and the Pacific region. Workshop to commemorate the international year of the potato. FAO.

Wingler, A., Lea, P.J., Quick, W.P. and Leegood, R.C. (2000) Photorespiration: metabolic pathways and their role in stress protection. Philos. Trans. R. Soc. Lond. B Biol. Sci. 355, 1517-1529.

Zhang, L., Häusler, R.E., Greiten, C., Hajirezaei, M.R., Haferkamp, I., Neuhaus, H.E., Flügge, U.I. and Ludewig, F. (2008) Overriding the co-limiting import of carbon and energy into tuber amyloplasts increases starch content and yield of transgenic potato plants. Plant Biotechnol. J. 6, 453-464.

Zrenner, R., Krause, K.P., Apel, P. and Sonnerwald, U. (1996) Reduction of the cytosolic fructose-1,6-bisphosphatase in transgenic potato plants limits photosynthetic sucrose biosynthesis with no impact on plant growth and tuber yield. Plant J. 9, 671-681.

\section{Supporting information}

Additional Supporting information may be found in the online version of this article:

Figure $\mathbf{S 1} \mathrm{A} / \mathrm{Ci}$-curves measured in transgenic plants.

Figure S2 Fumarase and catalase activity assays of the chloroplast fractions from wild-type and DEFp transgenics used for glycolate dehydrogenase activity analysis.

Table S1 Leaf metabolite contents of wild-type and DEFp transgenic potato plants. 